Research Paper

\title{
Correlation of PD-L1 and SOCS3 Co-expression with the Prognosis of Hepatocellular Carcinoma Patients
}

\author{
Liuxi Chen ${ }^{1,2^{*}}$, Xingxing Huang ${ }^{1,2^{*}}$, Wenzheng Zhang1,2, Ying Liu ${ }^{1,2}$, Bi Chen ${ }^{1,2,3}$, Yu Xiang ${ }^{2}$, Ruonan \\ Zhang1,2,3, Mingming Zhang ${ }^{2}$, Jiao Feng'2, Shuiping Liu'², Ting Duan², Xiaying Chen², Wengang Wang1,2, \\ Ting Pan², Lili Yan², Ting Jin², Guohua Li², Yongqiang Li ${ }^{1 凶}$, Tian Xie ${ }^{1,2,3 凶}$ and Xinbing Sui ${ }^{1,2,3 凶}$
}

1. Department of Medical Oncology, the Affiliated Hospital of Hangzhou Normal University, College of Medicine, Hangzhou Normal University, Hangzhou, Zhejiang, China.

2. Key Laboratory of Elemene Class Anti-cancer Chinese Medicine of Zhejiang Province, Hangzhou Normal University, Hangzhou, Zhejiang, China.

3. State Key Laboratory of Quality Research in Chinese Medicines, Faculty of Chinese Medicine, Macau University of Science and Technology, Macau, P.R. China.

*These authors contributed equally to this work.

$\triangle$ Corresponding authors: Xinbing Sui, E-mail: 20170022@hznu.edu.cn; Tian Xie, E-mail: xbs@dljg.sina.net or Yongqiang Li, E-mail: liyonq@hznu.edu.cn.

(c) The author(s). This is an open access article distributed under the terms of the Creative Commons Attribution License (https://creativecommons.org/licenses/by/4.0/). See http://ivyspring.com/terms for full terms and conditions.

Received: 2020.03.19; Accepted: 2020.06.14; Published: 2020.07.11

\begin{abstract}
Purpose: To investigate the correlation between the expression of PD-L1, SOCS3 and immune-related biomarkers CD276, CD4, CD8 in hepatocellular carcinoma (HCC) and further determine the relationship with clinicopathologic characteristics and the prognostic value of their co-expression in $\mathrm{HCC}$ patients.

Methods: We assessed the expression of PD-L1, CD276, SOCS3, CD4 and CD8 by immunohistochemistry in tumor tissue from $74 \mathrm{HCC}$ patients who underwent curative hepatectomy.

Results: High expression of PD-L1 was significantly associated with high Edmondson grade $(p<0.01)$ and elevated enzyme $(p=0.037)$; high expression of CD276 was significantly correlated with high Edmondson grade $(p=0.021)$; high expression of SOCS3 was significantly associated with age $(p=0.026)$ and tumor size $(p=0.041)$, while PD-L1 showed no significant correlation. The expression of PD-L1, CD276, SOCS3 protein and other clinicopathological factors (sex, vascular invasion, tumor number, tumor capsule, $\mathrm{PT}$ stage, liver cirrhosis, HBsAg, TBiL, AFP) showed no significant correlation $(p>0.05)$. High expression of CD8 was respectively significantly associated with worse overall survival $(O S)(p=0.002)$. There was no significantly difference between CD4 and CD8 high-expression and overall survival $(O S)(p=0.100)$. Both high expression of PD-L1 $(p=0.003)$ and low expression of SOCS3 $(p=0.015)$ was significantly associated with worse overall survival (OS). But CD276 only had a trendency $(p=0.166)$. Additionally, multivariate Cox regression models implied that PD-L1, SOCS3, as well as both CD4 and CD8 was an independent prognostic factor for OS $(\mathrm{p}<0.05)$. Furthermore, HCC patients with PD-L1 low-expression and SOCS3 high-expression had a better prognostic according to the different $p T$ stages $(p<0.05)$.

Conclusions: We for the first time demonstrated that PD-LI and SOCS3 were independent prognostic factor for HCC patients. Co-expression of low PD-L1 and high SOCS3 could be a better predictive marker for HCC patients.
\end{abstract}

Key words: PD-L1, SOCS3, CD276, CD4, CD8, hepatocellular carcinoma, prognostic value

\section{Introduction}

Hepatocellular carcinoma (HCC) remains one of the most common and aggressive malignancies worldwide [1]. It is reported that hepatocellular carcinoma has the fifth highest mortality rate in males and the sixth highest in females, due to the lack of effective diagnostic options and the late treatment [1, 2]. Incidence rates showed higher numbers in China mainly associated with chronic hepatitis B virus 
(HBV) infection [3, 4]. Recurrence, metastasis and frequent drug resistance are the main dilemma to the integrative cancer treatment of HCC [5]. Recently, immunotherapy has been attempted to further improve prognosis with advanced HCC [6]. By analyzing inflammatory gene including expression profiles, infiltrates, and regulatory molecules, an 'immune class' accounting for $30 \%$ of HCCs was characterized by immune cell infiltration, programmed cell death protein 1 (PD-1) and/or programmed cell death 1 ligand 1 (PD-L1) expression, IFN- $\gamma$ signaling activation and markers of cytolytic activity [7].

Programmed cell death protein 1 (PD-1) is a receptor mainly expressed by $\mathrm{T}$ cells, which negatively regulates the effector phase of $\mathrm{T}$ cell responses. To date, monoclonal antibodies to either PD-L1 (atezolizumab, avelumab, and durvalumab) or PD-1 (nivolumab and pembrolizumab) are approved for the cancer treatment [8]. PD-L1, as an independent predictive biomarker, is demonstrated low prediction accuracy attributed to the technical obstacles (variable detection antibodies, differing IHC cutoffs, staining of tumor versus immune cells) and the complexity of biological mechanisms (unevenly distribution, unstable expression) [9]. Therefore, we tried to explore potential biomarkers in combination to verify what prognostic clinical utility they may hold.

The PD-1/PD-L1 interactions suppress CD8 cytotoxic T lymphocyte (CTL) survival, proliferation and effector function and can also induce the apoptosis of tumor-infiltrating $\mathrm{T}$ cells [10]. PD-1/ PD-L1 interactions lead the differentiation of CD4 T cells to Foxp3 regulatory T cells (Tregs) [11], which are known to further inhibit the immune system and promote peripheral immune tolerance [12]. Tumorinfiltrating lymphocytes (TILs) are a group of lymphocytes located around tumor cells. The presence of TILs is associated with tumor regression and prognosis in HCC, which is often regarded as an "immunogenic tumor" [13].

B7-H3 (also known as CD276), a co-stimulator molecule of the cell surface B7 protein superfamily, is mainly expressed on dendritic cells along with $\mathrm{T}$ lymphocytes, B monocytes and NK cells [14]. It was reported that $\mathrm{CD} 276$ had a co-stimulating effect on the proliferation of both CD4 and CD8 T cells. Combinatorial blockade of anti-CD276 and anti-PD-1 has approved that this approach can be mutually enhancing treatment effect of established tumors [15]. Kang et al. have shown that down regulation of CD276 can significantly reduce cell migration and invasion in HCC, which may be referred to epithelialto-mesenchymal transition through the JAK2/ STAT3/Slug pathway [16].
Suppressor of cytokine signaling 3 (SOCS3) is a tumor suppressor gene and its inactivation may play a role in HCC initiation and development [17]. The deletion of the SOCS3 is an important mechanism for the activation of the STAT3 and this mechanism vitally act in the occurrence and progression of hepatitis-related liver cancer [18]. Zhang et al. found that overexpression of SOCS3 strongly inhibited STAT1 phosphorylation and PD-L1 up-regulation [19].

To our knowledge, PD-L1 is respectively related to $\mathrm{CD} 4, \mathrm{CD} 8, \mathrm{CD} 276$, SOCS3, but the relationship between those co-expression and hepatocellular carcinoma has not been reported. Hence, we retrospectively selected 74 hepatocellular carcinoma cases and performed immunohistochemical analysis for PD-L1, CD4, CD8, CD276 and SOCS3 for each paraffin-embedded block. In addition, we also investigated the correlation of the expression of CD4, CD8, PD-L1, CD276 and SOCS3 with clinicopathological features and clinical outcomes. We are committed to find potential biomarkers in combination with PD-L1 to predict hepatocellular carcinoma progression and prognosis.

\section{Materials and Methods}

\section{Public Gene Expression Omnibus dataset analysis}

Two publicly available Gene Expression Omnibus (GEO) datasets (GSE102079 and GSE121248) containing gene expression information from paracancerous tissues and cancerous tissues were downloaded from Gene Expression Omnibus (http://www.ncbi.nlm.nih.gov/geo/). The processed data dealed with normalization procedures were directly obtained from the corresponding websites, and no other transformations were performed.

\section{Patient population and tumor samples}

All patient information and clinical data were obtained from National Human Genetic Resources Sharing Service Platform. A total of 74 patients with formalin-fixed, paraffin-embedded tumors (FFPE) were selected retrospectively, who had undergone surgery without neoadjuvant treatment between June 2007 and March 2008. Patients were histopathologically diagnosed as hepatocellular carcinoma. Tumor staging and grading was carried out according to the American Joint Committee on Cancer (AJCC), TNM Staging System for Urethral Carcinoma (8th ed., 2017). When the patient died, the cause of death was determined by the attending physician or by death certificate. 


\section{Immunohistochemistry}

PD-L1, CD4, CD8, CD276 and SOCS3 immunohistochemistry (IHC) were available in 74 cases. Each case was performed PD-L1, CD4, CD8, CD276 and SOCS3 immunohistochemical staining using the same paraffin-embedded blocks. The sections were incubated with PD-L1 antibody (1:200 dilution; 13684S, Cell Signaling, USA), CD4 antibody (RMA-0620, Maixin Biotechnologies, China), CD8 antibody (MAB-0021, Maixin Biotechnologies, China), CD276 (1:400 dilution; HPA009285, Sigma, USA) and SOCS3 (1:500 dilution; ab16030, Abcam, UK) respectively overnight at $4{ }^{\circ} \mathrm{C}$. A DAB Kit (K0675, Dako, Denmark) was applied to the sections, followed by haematoxylin counterstain.

Comparing cancer tissue with paracancerous tissue, the staining conditions were established for each antibody, which were used as internal control in each staining protocol. In CD4/CD8 and CD276 IHC, cell membrane and cytoplasmic expression with higher intensity than internal positive control was regarded as positive. Cell membrane and cytoplasmic staining in 74 cases with higher intensity than internal positive control was interpreted as positive in PD-L1 or SOCS3 IHC. In each case, at least 500 cells were evaluated in 5 representative high power fields $(200 \times)$, and the proportion of positively stained cells were calculated.

According to the staining protocol, the staining conditions for each antibody were established and paracancerous tissue was used as internal control. Based on a four-point system, cytoplasmic/ membranous protein expression was scored by the staining intensity ( 0 : negative; 1 : weak; 2 : moderate; 3 : strong) and density (0: none; 1 : less than $40 \%$; 2 : $40-70 \%$; 3: more than 70\%). Quantitation of immunohistochemical staining was obtained by multiplying staining percentage by staining intensity, 0-3 were defined as low-expression whereas 4-9 were defined as high-expression. All immunostaining scores were obtained from five randomized fields by two investigators who were blinded to the clinical data.

\section{Statistical analyses}

Statistical analyses were conducted by SPSS software (version 23.0; IBM, Armonk, NY). Either Chi-square test or Fisher's exact test was used to explore the correlation between immunostaining markers and clinicopathological variables by Pearson's contingency analysis. The univariate Cox regression model was performed on the clinicopathological features and immunostaining markers as covariates. Multivariate Cox proportional-hazards analysis was conducted including the significant covariates ( $p$ value $<0.11$ ) according to the univariate analysis. Overall survival (OS) was from the date of curative hepatectomy to death. Kaplan-Meier analysis was used the log-rank test to compare differences. Two-tailed $\mathrm{p}$ value $<0.05$ was considered as statistically significant in all cases.

\section{Results}

\section{Expression of PD-L1, CD4, CD8, CD276 and SOCS3 in Hepatocellular Carcinoma}

To investigate the role of PD-L1, CD4, CD8, CD276 and SOCS3 in Hepatocellular Carcinoma, we processed GSE102079 and GSE121248 datasets from Gene Expression Omnibus (GEO). Both GSE102079 and GSE121248 datasets indicated that CD4 and SOCS3 expression levels of cancerous tissues were significantly downregulated compared with the paracancerous tissues and normal tissues $(\mathrm{P}<0.001$, Figure $1 \mathrm{Q}, \mathrm{R})$.

IHC was carried out in 74 specimens to examine the expression of PD-L1, CD4, CD8, CD276 and SOCS3 in HCC patients who underwent surgical treatment, which was selected regarding to the AJCC guideline (Table 1). In the HCC tissues, PD-L1 was mainly expressed in the cellular membrane and cytoplasm of tumor cells in a diffuse manner; CD4/ CD8, CD276 and SOCS3 protein were also mainly found in the cytoplasm and membrane of tumor cells (Figure 1). Twenty-six (35.1\%) of 74 tumors in hepatocellular carcinoma had elevated levels of PD-L1 protein as detected by IHC, whereas high PD-L1 expression significantly associated with low alanine transaminase (ALT) levels $(p=0.037)$ and high Edmondson grading $(\mathrm{P}<0.001)$ (Table 2$)$. However, no statistically significant correlation was observed between the expression of PD-L1 and other clinicopathological features. CD4 and CD8 exhibited high expression rates in the HCC tissue with values of $48.6 \%(36 / 74)$ and $54.1 \%(40 / 74)$, indicating that there were no significant association between the expression of CD4 and CD8 and other clinicopathological features (Table 1). Furthermore, CD276 high expression and SOCS3 low expression in HCC tissue were significantly associated with high Edmondson grading $(\mathrm{P}=0.021)$ and age, tumor size $(\mathrm{P}=0.026,0.041)$, respectively (Table 2$)$.

\section{Survival Analysis}

Kaplan-Meier survival and disease analysis of 74 HCC patients (Figure 1) showed significantly worse OS for the PD-L1 high-expression group compared with low-expression group $(\mathrm{P}=0.003)$. CD8 were significantly associated with survival from hepatocellular carcinoma $(p=0.002)$, while low- 
expression of CD4/CD8 just showed a trend towards patient survival $(p=0.100)$. Furthermore, the SOCS3 low-expression group had shorter OS than the corresponding high-expression group $(\mathrm{P}=0.015)$. However, no statistically significant association was observed between the expression of CD276 and OS. The followed-up period was 60 months or more, and 32 patients were dead at the time of analysis.

Table 1. Association of the expression of CD4 and CD8 with clinicopathologic characteristics of 74 hepatocellular carcinoma patients who underwent surgical treatment

\begin{tabular}{|c|c|c|c|c|c|c|c|c|}
\hline \multirow[t]{3}{*}{ Characteristic } & \multicolumn{2}{|c|}{ Patients } & \multicolumn{2}{|c|}{ CD4 Expression } & & \multicolumn{3}{|c|}{ CD8 Expression } \\
\hline & \multirow[t]{2}{*}{ No. } & \multirow[t]{2}{*}{$\%$} & \multicolumn{2}{|c|}{ High-expression } & \multirow[t]{2}{*}{$\mathrm{p}$-value } & \multicolumn{2}{|c|}{ High-expression } & \multirow[t]{2}{*}{ p-value } \\
\hline & & & No. & $\%$ & & No. & $\%$ & \\
\hline Total & 74 & 100 & 36 & 48.6 & & 40 & 54.1 & \\
\hline Gender & & & & & 0.621 & & & 0.283 \\
\hline Female & 6 & 8.1 & 4 & 66.7 & & 5 & 51.5 & \\
\hline Male & 68 & 91.9 & 32 & 47.1 & & 35 & 83.3 & \\
\hline Age & & & & & 0.106 & & & 0.448 \\
\hline$\leq 52$ & 40 & 54.1 & 20 & 50.0 & & 20 & 50.0 & \\
\hline$>52$ & 34 & 45.9 & 16 & 47.1 & & 20 & 58.8 & \\
\hline \multicolumn{3}{|c|}{ Edmondson grading } & & & 0.990 & & & 0.613 \\
\hline I-II & 35 & 47.3 & 17 & 48.6 & & 20 & 57.1 & \\
\hline III-IV & 39 & 52.7 & 19 & 48.7 & & 20 & 51.3 & \\
\hline \multicolumn{3}{|c|}{ Vessel invasion } & & & 0.232 & & & 0.498 \\
\hline Yes & 19 & 25.7 & 7 & 36.8 & & 9 & 47.4 & \\
\hline No & 55 & 74.3 & 29 & 52.7 & & 31 & 56.4 & \\
\hline Tumor size & & & & & 0.737 & & & 0.326 \\
\hline$>5 \mathrm{~cm}$ & 24 & 32.4 & 11 & 45.8 & & 11 & 45.8 & \\
\hline$\leq 5 \mathrm{~cm}$ & 50 & 67.6 & 25 & 50.0 & & 29 & 58.0 & \\
\hline \multicolumn{3}{|l|}{ Tumor number } & & & 0.945 & & & 0.525 \\
\hline 1 & 68 & 91.9 & 33 & 48.5 & & 38 & 55.9 & \\
\hline 2 & 6 & 8.1 & 3 & 50.0 & & 2 & 33.3 & \\
\hline \multicolumn{3}{|l|}{ Tumor capsule } & & & 0.153 & & & 0.138 \\
\hline Complete & 33 & 44.6 & 13 & 39.4 & & 21 & 63.6 & \\
\hline Incomplete & 41 & 55.4 & 23 & 56.1 & & 19 & 46.3 & \\
\hline \multicolumn{2}{|l|}{ pT categories } & & & & 0.288 & & & 0.215 \\
\hline pT1 & 49 & 66.2 & 26 & 53.1 & & 29 & 59.2 & \\
\hline $\mathrm{pT} 2 / \mathrm{pT} 3$ & 25 & 33.8 & 10 & 40.0 & & 11 & 44.0 & \\
\hline \multicolumn{2}{|l|}{ Cirrhosis } & & & & 0.769 & & & 0.808 \\
\hline Positive & 66 & 89.2 & 33 & 50.0 & & 36 & 54.5 & \\
\hline Negative & 8 & 10.8 & 3 & 37.5 & & 4 & 50.0 & \\
\hline \multicolumn{2}{|l|}{ HBsAg } & & & & 0.687 & & & 0.315 \\
\hline Positive & 57 & 77.0 & 27 & 47.4 & & 29 & 50.9 & \\
\hline Negative & 17 & 23.0 & 9 & 52.9 & & 11 & 64.7 & \\
\hline \multicolumn{2}{|l|}{ TBiL } & & & & 0.418 & & & 0.987 \\
\hline 3.5-20umol/L & 61 & 82.4 & 31 & 50.8 & & 33 & 54.1 & \\
\hline$>20 \mathrm{umol} / \mathrm{L}$ & 13 & 17.6 & 5 & 38.5 & & 7 & 53.8 & \\
\hline \multicolumn{2}{|l|}{ ALT } & & & & 0.790 & & & 0.121 \\
\hline $0-40 \mathrm{U} / \mathrm{L}$ & 42 & 56.8 & 21 & 50.0 & & 26 & 61.9 & \\
\hline$>40 \mathrm{U} / \mathrm{L}$ & 32 & 43.2 & 15 & 46.9 & & 14 & 43.8 & \\
\hline \multicolumn{2}{|l|}{ AFP } & & & & 0.247 & & & 0.801 \\
\hline$<100 u g / L$ & 38 & 51.4 & 16 & 42.1 & & 20 & 52.6 & \\
\hline$\geq 100 \mathrm{ug} / \mathrm{L}$ & 36 & 48.6 & 20 & 55.6 & & 20 & 55.6 & \\
\hline
\end{tabular}

Based on these results, we divided the cohort into two groups (49 for pT1 and 25 for pT2/pT3) for further Kaplan-Meier analysis (Figure 2). Combination CD4 low-expression with CD8 lowexpression versus the others were significantly association with survival for pT2/pT3 $(\mathrm{P}=0.025)$, whereas no statistically significant association was observed for pT1. Patients in PD-L1 low-expression and SOCS3 high-expression both had better OS
$(\mathrm{P}=0.012, \mathrm{P}=0.024)$ than the other group for $\mathrm{pT} 1$ group. And for $\mathrm{pT} 2 / \mathrm{pT} 3$ group, PD-L1 were also associated with hepatocellular carcinoma survival $(\mathrm{P}=0.049)$. However, OS about the SOCS3 highexpression group had no significant association with the corresponding low-expression group for pT2/ pT3.

According to the results, we combined PD-L1 with SOCS3, and stratified as four types (both high-expression, both low-expression, high-/lowexpression, low-/high-expression). Patients could be divided into low- and high-risk for death. Of the 49 patients, 20 of them were PD-L1 low-expression and SOCS3 high-expression status, while the rest with the other three groups. When analyzed as categoric variables in Kaplan-Meier analysis, the hepatocellular carcinoma had a better prognosis $(\mathrm{P}=0.006)$. There was a similar relationship for $\mathrm{pT} 2 / \mathrm{pT} 3$ group between PD-L1 and SOCS3 staining $(\mathrm{P}=0.043)$ (Figure 2).

\section{Cox Analysis}

Given the significant correlation between the expression of CD4/CD8, PD-L1, CD276 and SOCS3, we performed univariate and multivariate Cox proportional-hazards analyses. Univariate analysis (Table 3) revealed that Edmondson grading $(\mathrm{P}=0.005)$, tumor number $(\mathrm{P}=0.004)$, $\mathrm{pT}$ categories $(\mathrm{P}=0.032)$, PD-L1 expression $(\mathrm{P}=0.004)$ and SOCS3 expression $(\mathrm{P}=0.019)$ were independent indicators for overall survival. We also confirmed that the tumor size $(\mathrm{P}=0.096)$, tumor capsule $(\mathrm{P}=0.083)$ and $\mathrm{CD} 4 / \mathrm{CD} 8$ expression $(\mathrm{P}=0.107)$ have the trend with HCC survival. According to the data above, multivariate Cox regression model (Table 4), including the covariates of pT categories (pT1 vs. PT2/pT3), Edmondson grading (I-II vs. III-IV), tumor capsule (complete vs. incomplete), co-overexpression of both CD4 and CD8, PD-L1 expression and SOCS3 expression, exhibited that pT categories $(\mathrm{P}=0.045)$, CD4/CD8 expression $(\mathrm{P}=0.040)$, $\mathrm{PD}-\mathrm{L} 1$ expression $(\mathrm{P}<0.001)$ and SOCS3 expression $(\mathrm{P}<0.001)$ were independent predictive factors for OS.

\section{Discussion}

Hepatocellular carcinoma is prone to recurrence; metastasis and drug resistance may be the main obstacles in clinical management [5]. Immunotherapy is attractive for hepatocellular carcinoma, which is considered as an "immunogenic tumor" [7]. Moreover, tumor-infiltrating lymphocytes are crucial components of immune cell in tumor microenvironment which participate in tumorigenesis, tumor progression and play the key role in anti-tumor immune therapy [20]. PD-1 is 
defined as a negative molecular of the immune response and primarily expressed on activated $\mathrm{T}$ and B cells [21]. PD-1/PD-L1 pathway is an important mechanism of immunosuppression in TME [21]. CD276, as a costimulatory molecule, is participate in the secretion of IFN- $\gamma$ during the T cell activation, and also regulates tumor immune microenvironment involving the aggressiveness of various tumors [22-24]. The down-regulation of CD276 may be related to the JAK/STAT3 pathway in HCC [16]. And the JAK/STAT3 pathway is the canonical target of SOCS3 expression, which is regarded as a tumor suppressor gene [18]. The activation of STAT3 in hepatocytes is mediated via deletion of the SOCS3, resulting in hepatocarcinogenesis [18].

In the present study, we demonstrated that early-stage HCC patients with down regulation of CD8 in tumor tissue had a significantly higher risk of death, whereas no significant difference was observed in CD4 expression. These results further support that CD8 T lymphocytes play a vital role in antitumor immune response [25].

Table 2. Association of the expression of PD-L1, CD276 and SOCS3 with clinicopathologic characteristics of 74 hepatocellular carcinoma patients who underwent surgical treatment

\begin{tabular}{|c|c|c|c|c|c|c|c|c|c|c|c|}
\hline \multirow[t]{3}{*}{ Characteristic } & \multicolumn{2}{|c|}{ Patients } & \multicolumn{3}{|c|}{ PD-L1 Expression } & \multicolumn{3}{|c|}{ CD276 Expression } & \multicolumn{3}{|c|}{ SOCS3 Expression } \\
\hline & \multirow[t]{2}{*}{ No. } & \multirow[t]{2}{*}{$\%$} & \multicolumn{2}{|c|}{ High-expression } & \multirow[t]{2}{*}{ p-value } & \multicolumn{2}{|c|}{ High-expression } & \multirow[t]{2}{*}{ p-value } & \multicolumn{2}{|c|}{ High-expression } & \multirow[t]{2}{*}{ p-value } \\
\hline & & & No. & $\%$ & & No. & $\%$ & & No. & $\%$ & \\
\hline Total & 74 & 100 & 26 & 35.1 & & 38 & 51.4 & & 49 & 66.2 & \\
\hline Gender & & & & & 0.923 & & & 0.178 & & & 0.981 \\
\hline Female & 6 & 8.1 & 2 & 33.3 & & 1 & 16.7 & & 4 & 66.7 & \\
\hline Male & 68 & 91.9 & 24 & 35.3 & & 37 & 54.4 & & 45 & 66.2 & \\
\hline Age & & & & & 0.644 & & & 0.801 & & & $0.026^{*}$ \\
\hline$\leq 52$ & 40 & 54.1 & 15 & 37.5 & & 20 & 50.0 & & 31 & 77.5 & \\
\hline$>52$ & 34 & 45.9 & 11 & 32.4 & & 18 & 52.9 & & 18 & 52.9 & \\
\hline Edmondson grading & & & & & $<0.001^{*}$ & & & $0.021^{*}$ & & & 0.685 \\
\hline I-II & 35 & 47.3 & 5 & 14.3 & & 13 & 37.1 & & 24 & 68.6 & \\
\hline III-IV & 39 & 52.7 & 21 & 53.8 & & 25 & 64.1 & & 25 & 64.1 & \\
\hline Vessel invasion & & & & & 0.350 & & & 0.897 & & & 0.374 \\
\hline Yes & 19 & 25.7 & 5 & 26.3 & & 10 & 52.6 & & 11 & 57.8 & \\
\hline No & 55 & 74.3 & 21 & 38.2 & & 28 & 50.9 & & 38 & 69.1 & \\
\hline Tumor size & & & & & 0.822 & & & 0.405 & & & $0.041^{*}$ \\
\hline$>5 \mathrm{~cm}$ & 24 & 32.4 & 8 & 33.3 & & 14 & 58.3 & & 12 & 50.0 & \\
\hline$\leq 5 \mathrm{~cm}$ & 50 & 67.6 & 18 & 36.0 & & 24 & 48.0 & & 37 & 74.0 & \\
\hline Tumor number & & & & & 0.214 & & & 0.227 & & & 0.635 \\
\hline 1 & 68 & 91.9 & 22 & 32.4 & & 33 & 48.5 & & 44 & 64.7 & \\
\hline 2 & 6 & 8.1 & 4 & 66.7 & & 5 & 83.3 & & 5 & 83.3 & \\
\hline Tumor capsule & & & & & 0.078 & & & 0.658 & & & 0.360 \\
\hline Complete & 33 & 44.6 & 8 & 24.2 & & 16 & 48.5 & & 20 & 60.6 & \\
\hline Incomplete & 41 & 55.4 & 18 & 43.9 & & 22 & 53.7 & & 29 & 70.7 & \\
\hline pT categories & & & & & 0.911 & & & 0.288 & & & 0.773 \\
\hline pT1 & 49 & 66.2 & 17 & 34.7 & & 23 & 46.9 & & 33 & 67.3 & \\
\hline $\mathrm{pT} 2 / \mathrm{pT} 3$ & 25 & 33.8 & 9 & 36.0 & & 15 & 60.0 & & 16 & 64.0 & \\
\hline Cirrhosis & & & & & 0.304 & & & 0.228 & & & 0.341 \\
\hline Positive & 66 & 89.2 & 25 & 37.9 & & 36 & 54.5 & & 42 & 63.6 & \\
\hline Negative & 8 & 10.8 & 1 & 12.5 & & 2 & 25.0 & & 7 & 87.5 & \\
\hline HBsAg & & & & & 0.241 & & & 0.881 & & & 0.187 \\
\hline Positive & 57 & 77.0 & 18 & 31.6 & & 29 & 50.9 & & 40 & 70.2 & \\
\hline Negative & 17 & 23.0 & 8 & 47.1 & & 9 & 52.9 & & 9 & 52.9 & \\
\hline TBiL & & & & & 0.186 & & & 0.102 & & & 0.800 \\
\hline 3.5-20 umol/L & 61 & 82.4 & 24 & 39.3 & & 34 & 55.7 & & 40 & 65.6 & \\
\hline$>20 \mathrm{umol} / \mathrm{L}$ & 13 & 17.6 & 2 & 15.4 & & 4 & 30.8 & & 9 & 69.2 & \\
\hline ALT & & & & & $0.037^{*}$ & & & 0.107 & & & 0.555 \\
\hline $0-40 \mathrm{U} / \mathrm{L}$ & 42 & 56.8 & 19 & 45.2 & & 25 & 59.5 & & 29 & 69.0 & \\
\hline$>40 \mathrm{U} / \mathrm{L}$ & 32 & 43.2 & 7 & 21.9 & & 13 & 40.6 & & 20 & 62.5 & \\
\hline AFP & & & & & 0.103 & & & 0.481 & & & 0.936 \\
\hline$<100 \mathrm{ug} / \mathrm{L}$ & 38 & 51.4 & 10 & 26.3 & & 18 & 47.4 & & 25 & 65.8 & \\
\hline$\geq 100 \mathrm{ug} / \mathrm{L}$ & 36 & 48.6 & 16 & 44.4 & & 20 & 55.6 & & 24 & 66.7 & \\
\hline CD4 expression & & & & & 0.422 & & & 0.481 & & & 0.059 \\
\hline Low & 38 & 51.4 & 15 & 39.5 & & 18 & 47.4 & & 29 & 76.3 & \\
\hline High & 36 & 48.6 & 11 & 30.6 & & 20 & 55.6 & & 20 & 55.6 & \\
\hline CD8 expression & & & & & 0.644 & & & 0.801 & & & 0.083 \\
\hline Low & 34 & 45.9 & 11 & 32.4 & & 18 & 52.9 & & 19 & 55.9 & \\
\hline High & 40 & 54.1 & 15 & 37.5 & & 20 & 50.0 & & 30 & 75.0 & \\
\hline CD4/CD8 expression & & & & & 0.966 & & & 0.684 & & & 0.683 \\
\hline Low & 23 & 31.1 & 8 & 34.8 & & 11 & 47.8 & & 16 & 69.6 & \\
\hline High & 51 & 68.9 & 18 & 24.3 & & 27 & 52.9 & & 33 & 64.7 & \\
\hline
\end{tabular}

*p-value $<0.05$. 


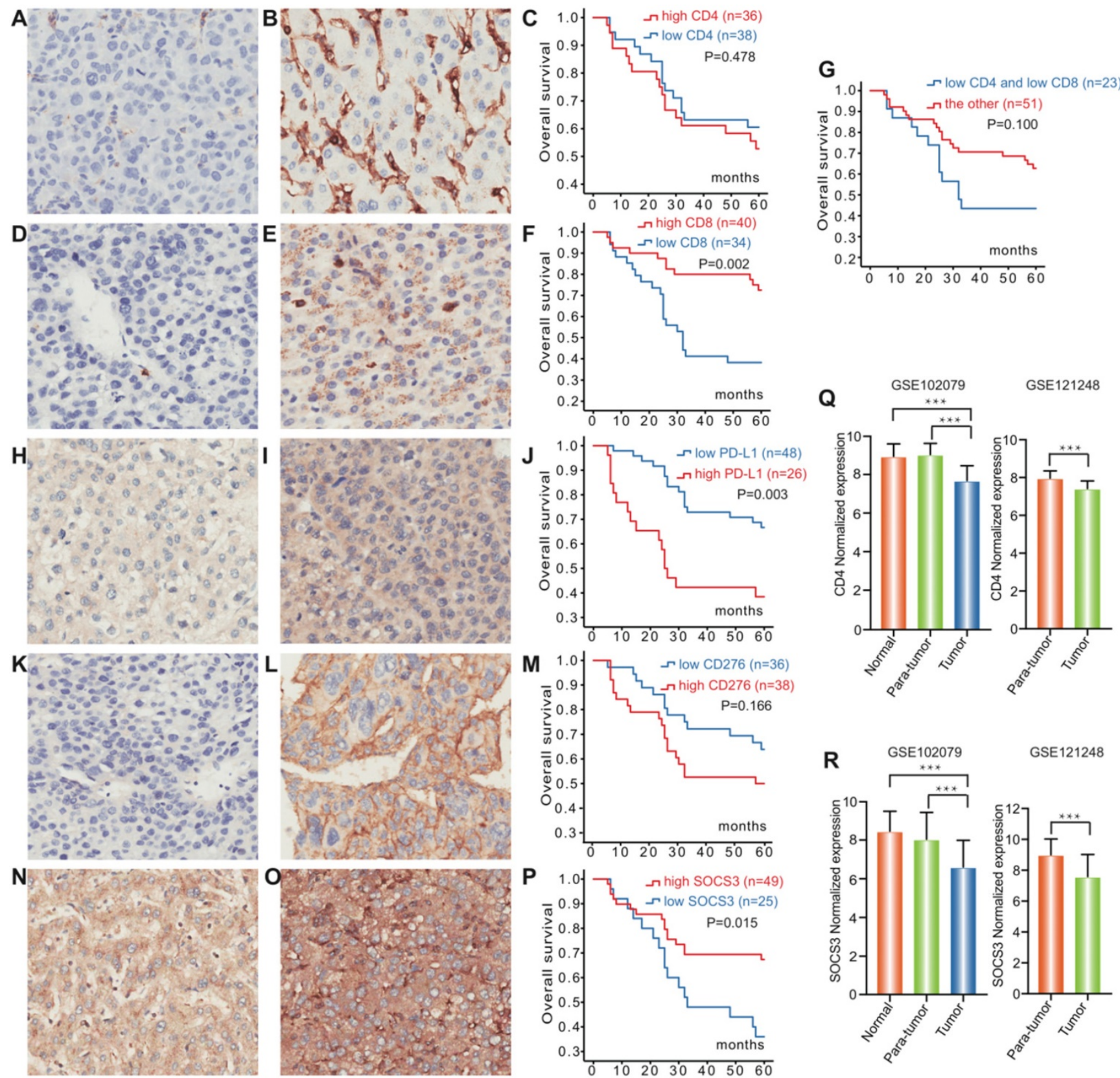

Figure 1. Expression of PD-L1, CD4, CD8, CD276 and SOCS3 in hepatocellular carcinoma. Representative immunohistochemical staining and Kaplan-Meier survival curves for CD4, CD8, PD-L1, CD276 and SOCS3 in hepatocellular Carcinoma. (A) CD4 low-expression; (B) CD4 high-expression; (C) No significant difference between the overall survival rate for patients with CD4 high-expression and that for patients with CD4 low-expression ( $=0.478)$; (D) $C D 8$ low-expression; (E) CD8 high- expression; (F) The overall survival rate for patients with CD8 high-expression was significantly higher than that for patients with $C D 8$ low-expression ( $P=0.002)$; (G) No significant difference between the overall survival rate for patients with CD4/CD8 low-expression and that for patients with the other ( $\mathrm{p}=0.100)$; $(\mathbf{H})$ PD-L1 low-expression; (I) PD-L1 high-expression; (J) The overall survival rate for patients with PD-L1 low-expression was significantly higher than that for patients with PD-L1 high-expression ( $\mathrm{P}=0.003$ ); $(\mathbf{K})$ CD276 low-expression; (L) CD276 high-expression; (M) No significant difference between the overall survival rate for patients with CD276 high-expression and that for patients with CD276 low-expression ( $p=0.166)$; $(\mathbf{N})$ SOCS3 low-expression; $(\mathbf{O})$ SOCS3 high-expression; $(\mathbf{P})$ The overall survival rate for patients with SOCS3 high-expression was significantly higher than that for patients with SOCS3 low-expression $(p=0.015)$. CD4 and SOCS3 expression statuses in hepatocellular carcinoma tissues and paracancerous tissues were obtained from GSE102079 and GSE121248. (Q) CD4 expression levels of cancerous tissues were significantly downregulated compared with the paracancerous and normal tissues $(\mathrm{P}<0.001)$; $(\mathbf{R})$ SOCS3 expression levels of cancerous tissues were significantly downregulated compared with the paracancerous and normal tissues $(\mathrm{P}<0.001)$.

Given the complexity of the interaction between CD4 T lymphocytes and target antigens, depletion of CD4 T lymphocytes could decrease antitumor activity [26]. We also verified that co-overexpression of
CD4/CD8 significantly improved the prognosis of HCC patients, and COX analysis further supported it as an independent prognostic factor for OS [26, 27]. 

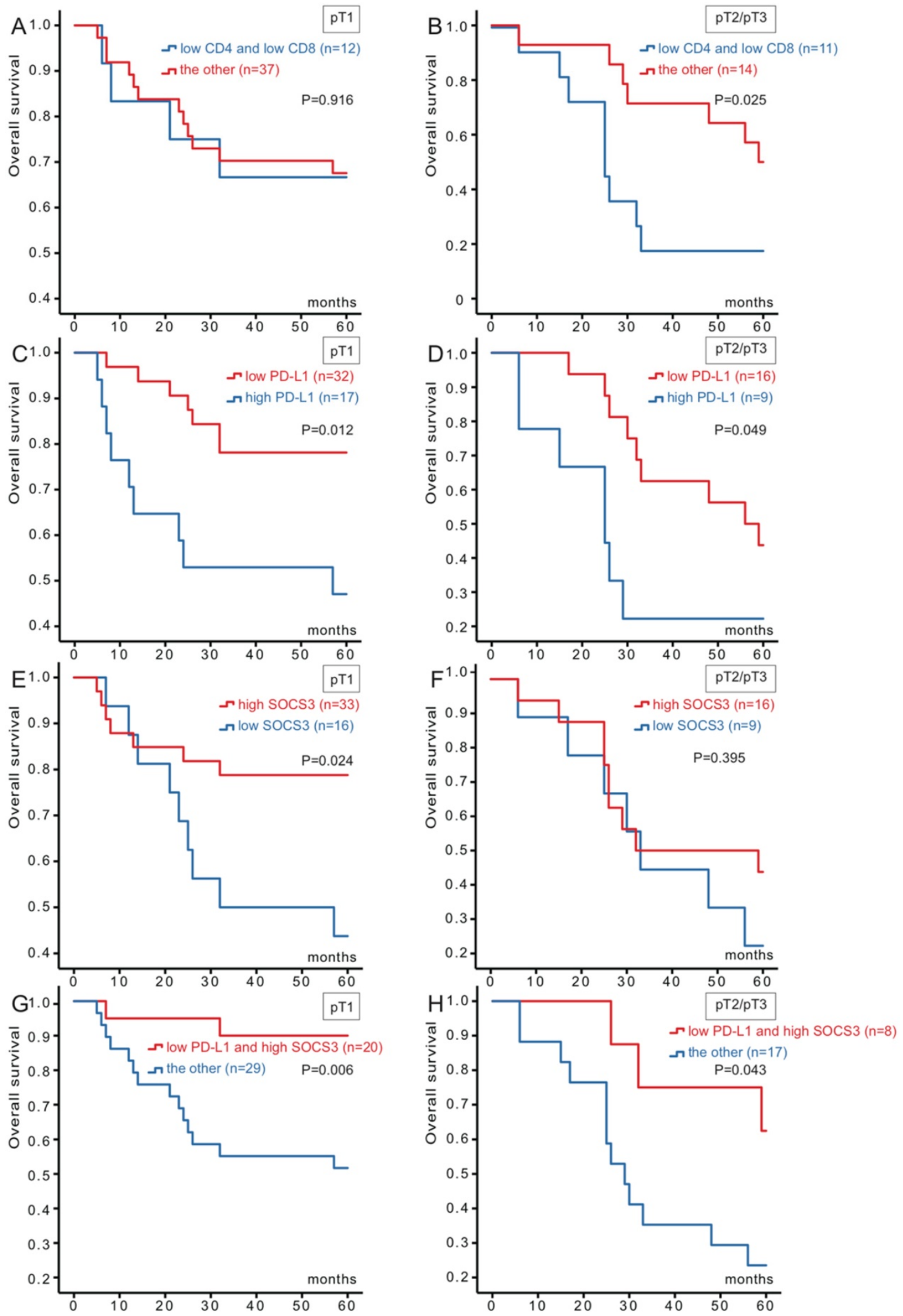

Figure 2. Kaplan-Meier survival curves of 74 hepatocellular carcinoma patients in different pT stages according to CD4/CD8, PD-L1 and SOCS3 expression. (A) No significant difference between the overall survival rate for patients with CD4/CD8 low-expression and that for patients with the other in PT1 stage $(p=0.916) ;(B)$ The overall survival rate for patients with $C D 4 / C D 8$ low-expression was significantly lower than that for patients with the other in $p T 2 / p T 3$ stage $(p=0.025) ;(C)$ The overall survival rate for patients with PD-L1 low-expression was significantly higher than that for patients with PD-L1 high-expression in $\mathrm{pT} 1$ stage $(\mathrm{p}=0.012) ;(\mathrm{D})$ The overall survival rate for patients with PD-L1 low-expression was significantly higher than that for patients with PD-L1 high-expression in $\mathrm{PT} 2 / \mathrm{PT}$ stage $(\mathrm{P}=0.049) ;(\mathrm{E})$ The overall survival rate for patients with SOCS3 high-expression was significantly higher than that for patients with SOCS3 low-expression in $\mathrm{p} T 1$ stage $(\mathrm{p}=0.024) ;(\mathbf{F})$ No significant difference between the overall survival rate for patients with SOCS3 high-expression and that for patients with SOCS3 low-expression in $\mathrm{PT} 2 / \mathrm{pT} 3$ stage $(\mathrm{p}=0.395)$; (G) The overall survival rate for patients with PD-L1 low-expression/SOCS3 high-expression was significantly higher than that for patients with the other in $\mathrm{pT} 1$ stage $(\mathrm{p}=0.006)$; $(\mathbf{H})$ The overall survival rate for patients with PD-L1 low-expression/SOCS3 high-expression was significantly higher than that for patients with the other in $\mathrm{PT} 2 / \mathrm{pT} 3$ stage $(\mathrm{p}=0.043)$. 
Table 3. Univariate cox regression analyses of pathologic features and molecular markers for the overall survival of 74 hepatocellular carcinoma patients who underwent surgical treatment

\begin{tabular}{llll}
\hline Variables & \multicolumn{3}{l}{ Univariate } \\
\cline { 2 - 4 } & Hazard Ratio & 95\%CI & p-value \\
\hline Gender (female vs. male) & 0.336 & $0.046-2.466$ & 0.284 \\
Age (<52y vs. $\geq 52$ y) & 0.606 & $0.301-1.219$ & 0.160 \\
Edmondson grading (I-II vs. III-IV) & 3.029 & $1.398-6.563$ & $0.005^{*}$ \\
Vessel invasion (positive vs. negative) & 1.296 & $0.613-2.739$ & 0.497 \\
Tumor size ( 5 cm vs. >5cm) & 1.812 & $0.900-3.648$ & 0.096 \\
Tumor number (1 vs. 2) & 3.705 & $1.501-9.144$ & $0.004^{*}$ \\
Tumor capsule (complete vs. incomplete) & 1.908 & $0.919-3.961$ & 0.083 \\
pT categories (pT1 vs. pT2/pT3) & 2.139 & $1.066-4.289$ & $0.032^{*}$ \\
Cirrhosis (positive vs. negative) & 2.046 & $0.489-8.568$ & 0.327 \\
HBsAg (positive vs. negative) & 1.142 & $0.513-2.542$ & 0.745 \\
TBiL (3.5-20umol/L vs. >20umol/L) & 1.213 & $0.467-3.151$ & 0.691 \\
ALT (0-40U/L vs. >40U/L) & 1.220 & $0.609-2.445$ & 0.574 \\
AFP (<100ug/L vs. $\geq 100 u g / L)$ & 1.365 & $0.681-2.735$ & 0.380 \\
CD4/CD8 expression (high vs. low) & 0.559 & $0.276-1.135$ & 0.107 \\
PD-L1 expression (high vs. low) & 2.754 & $1.373-5.525$ & $0.004^{*}$ \\
CD276 expression (high vs. low) & 1.633 & $0.806-3.309$ & 0.174 \\
SOCS3 expression (high vs. low) & 0.435 & $0.217-0.872$ & $0.019^{*}$ \\
\hline
\end{tabular}

${ }^{*}$ p-value $<0.05$

Table 4. Multivariate cox regression analyses of pathologic features and molecular markers for the overall survival of 74 hepatocellular carcinoma patients who underwent surgical treatment

\begin{tabular}{llll}
\hline Variables & \multicolumn{3}{l}{ Multivariate } \\
\cline { 2 - 4 } & Hazard Ratio & 95\%CI & p-value \\
\hline pT categories (pT1 vs. pT2/pT3) & 2.122 & $1.108-4.379$ & $0.045^{*}$ \\
Edmondson grading (I-II vs. III-IV) & 1.718 & $0.742-3.980$ & 0.207 \\
Tumor capsule (complete vs. incomplete) & 1.153 & $0.501-2.652$ & 0.737 \\
CD4/CD8 expression (high vs. low) & 0.455 & $0.214-0.966$ & $0.040^{*}$ \\
PD-L1 expression (high vs. low) & 5.275 & $2.506-13.082$ & $<0.001^{*}$ \\
SOCS3 expression (high vs. low) & 0.210 & $0.093-0.475$ & $<0.001^{*}$ \\
\hline
\end{tabular}

*p-value $<0.05$.

Patients with high PD-L1 expression or low SOCS3 expression had significantly poorer prognosis, which were suggested as independent prognostic factors. Further study had shown that PD-L1 IHC had a good prognostic value in different stages of HCC patients, while SOCS3 only associated with stage I patients, and had no significant impact in stage II disease. The PD-L1 expression alone does not sufficiently predict for response in HCC patients [28]. Therefore, a series of clinical trials has shown that PD-1/PD-L1 antibodies have promising outcomes for cancer treatment, only a part of patients responded to the treatments. It is noteworthy that only the combination of different markers can properly predict overall survival in HCC patients. We first found a significant correlation between the expression of PD-L1 and SOCS3 in HCC tumor tissue. High SOCS3 and low PD-L1 expressions had a better prognosis compared with the other. Additionally, the co-expression of SOCS3 and PD-L1 in HCC tissue had a best prognostic value in different $\mathrm{pT}$ stages compared with the individual markers. Recently, a series of studies have demonstrated that SOCS3 can also participate in the immune response by inhibiting the expression of inflammation-related cytokines [19, $29,30]$. It is striking that overexpression of SOCS3 strongly inhibited STAT1 phosphorylation and PD-L1 up-regulation, and further studies confirmed that SOCS3 regulates JAK/STAT3 pathway through immunoregulation $[19,31]$. As our results suggested that PD-L1 and SOCS3 are negatively correlated in HCC tissues, and it still remains ambiguous whether SOCS3 can selectively down-regulate PD-L1 to promote anti-cancer immune responses in HCC. Relevant experiments need to be designed to verify our speculation for HCC patients in the future. However, no significant correlation was found between the expression of CD276 and HCC tumor tissues in this study. The main reason is that CD276 has a dual role in tumor cells by interacting with T-suppressor cells or T-effector cells [32]. Future research needs to address the conundrum of CD276 and the sequence of its role in HCC.

In conclusion, we first reported the negative correlation between the expression of PD-L1 and SOCS3 in HCC tissue. At the same time, PD-L1 and SOCS3 were found to be independent prognostic factors for OS, and patients with PD-L1 low-expression and SOCS3 high-expression had the best prognosis compared with the individual markers in different $\mathrm{pT}$ stages. Therefore, frequent follow-up is needed for more patients with co-expression of PD-L1 and SOCS3. In addition, a combinational therapy with SOCS3 stimulator and PD-L1 blockade may be beneficial for HCC patients and needs to be examined in future studies.

\section{Abbreviations}

HCC: hepatocellular carcinoma; HBV: hepatitis B virus; PD-1: programmed cell death protein 1; PD-L1: programmed cell death 1 ligand 1; PD-L2: programmed cell death protein ligand 2; TME: tumor microenvironment; CTL: cytotoxic $\mathrm{T}$ lymphocyte; Tregs: regulatory $\mathrm{T}$ cells; TILs: tumor-infiltrating lymphocytes; TCR: T cell receptor; SOCS3: suppressor of cytokine signaling 3; GEO: gene expression omnibus; FFPE: formalin-fixed: paraffin-embedded tumors; AJCC: American Joint Committee on Cancer; IHC: immunohistochemistry; OS: overall survival.

\section{Acknowledgments}

This work was supported by grants from the National Natural Science Foundation of China (grant No. 81672932, 81730108, 81874380 and 81973635), Zhejiang Provincial Natural Science Foundation of China for Distinguished Young Scholars (grant No. LR18H160001), Zhejiang province science and 
technology project of TCM (grant No. 2019ZZ016, 2020ZQ046).

\section{Competing Interests}

The authors have declared that no competing interest exists. None of the contents of this manuscript has been previously published or is under consideration elsewhere. All the authors read and approved the final version of the manuscript prior to submission.

\section{References}

1. Siegel RL, Miller KD, Jemal A. Cancer statistics, 2020. CA Cancer J Clin. 2020; 70: 7-30.

2. Bray F, Ferlay J, Soerjomataram I, Siegel RL, Torre LA, Jemal A. Global cancer statistics 2018: GLOBOCAN estimates of incidence and mortality worldwide for 36 cancers in 185 countries. CA Cancer J Clin. 2018; 68: 394-424.

3. Schweitzer A, Horn J, Mikolajczyk RT, Krause G, Ott JJ. Estimations of worldwide prevalence of chronic hepatitis B virus infection: a systematic review of data published between 1965 and 2013. Lancet. 2015; 386: 1546-55.

4. Forner A, Reig M, Bruix J. Hepatocellular carcinoma. Lancet. 2018; 391: 1301-14.

5. Villanueva A, Llovet JM. Targeted therapies for hepatocellular carcinoma. Gastroenterology. 2011; 140: 1410-26.

6. El-Khoueiry AB, Sangro B, Yau T, Crocenzi TS, Kudo M, Hsu C, et al. Nivolumab in patients with advanced hepatocellular carcinoma (CheckMate 040): an open-label, non-comparative, phase $1 / 2$ dose escalation and expansion trial. Lancet. 2017; 389: 2492-502.

7. Sia D, Jiao Y, Martinez-Quetglas I, Kuchuk O, Villacorta-Martin C, Castro de Moura M, et al. Identification of an Immune-specific Class of Hepatocellular Carcinoma, Based on Molecular Features. Gastroenterology. 2017; 153: 812-26.

8. Sharma P, Hu-Lieskovan S, Wargo JA, Ribas A. Primary, Adaptive, and Acquired Resistance to Cancer Immunotherapy. Cell. 2017; 168: 707-23.

9. Cristescu R, Mogg R, Ayers M, Albright A, Murphy E, Yearley J, et al. Pan-tumor genomic biomarkers for PD-1 checkpoint blockade-based immunotherapy. Science. 2018; 362

10. Barber DL, Wherry EJ, Masopust D, Zhu B, Allison JP, Sharpe AH, et al. Restoring function in exhausted CD8 $\mathrm{T}$ cells during chronic viral infection. Nature. 2006; 439: 682-7.

11. Francisco LM, Salinas VH, Brown KE, Vanguri VK, Freeman GJ, Kuchroo VK, et al. PD-L1 regulates the development, maintenance, and function of induced regulatory T cells. J Exp Med. 2009; 206: 3015-29.

12. Adeegbe DO, Nishikawa $\mathrm{H}$. Natural and induced $\mathrm{T}$ regulatory cells in cancer. Front Immunol. 2013; 4: 190.

13. Miamen AG, Dong $\mathrm{H}$, Roberts LR. Immunotherapeutic approaches to hepatocellular carcinoma treatment. Liver Cancer. 2012; 1: 226-37.

14. Steinberger P, Majdic O, Derdak SV, Pfistershammer K, Kirchberger S, Klauser C, et al. Molecular characterization of human 4Ig-B7-H3, a member of the B7 family with four Ig-like domains. J Immunol. 2004; 172: 2352-9.

15. Yonesaka K, Haratani K, Takamura S, Sakai H, Kato R, Takegawa N, et al. B7-H3 Negatively Modulates CTL-Mediated Cancer Immunity. Clin Cancer Res. 2018; 24: 2653-64.

16. Kang FB, Wang L, Jia HC, Li D, Li HJ, Zhang YG, et al. B7-H3 promotes aggression and invasion of hepatocellular carcinoma by targeting epithelial-to-mesenchymal transition via JAK2/STAT3/Slug signaling pathway. Cancer Cell Int. 2015; 15: 45.

17. He B, You L, Uematsu K, Zang K, Xu Z, Lee AY, et al. SOCS-3 is frequently silenced by hypermethylation and suppresses cell growth in human lung cancer. Proc Natl Acad Sci U S A. 2003; 100: 14133-8.

18. Ogata H, Kobayashi T, Chinen T, Takaki H, Sanada T, Minoda Y, et al. Deletion of the SOCS3 gene in liver parenchymal cells promotes hepatitis-induced hepatocarcinogenesis. Gastroenterology. 2006; 131: 179-93.

19. Zhang Y, Ma CA, Lawrence MG, Break TJ, O'Connell MP, Lyons JJ, et al. PD-L1 up-regulation restrains Th17 cell differentiation in STAT3 loss- and STAT1 gain-of-function patients. J Exp Med. 2017; 214: 2523-33.

20. Kudo M. Immuno-Oncology in Hepatocellular Carcinoma: 2017 Update. Oncology. 2017; 93 Suppl 1: 147-59.

21. Topalian SL, Drake CG, Pardoll DM. Targeting the PD-1/B7-H1(PD-L1) pathway to activate anti-tumor immunity. Curr Opin Immunol. 2012; 24: 207-12.

22. Luo L, Chapoval AI, Flies DB, Zhu G, Hirano F, Wang S, et al. B7-H3 enhances tumor immunity in vivo by costimulating rapid clonal expansion of antigen-specific CD8+ cytolytic T cells. J Immunol. 2004; 173: 5445-50.

23. Chapoval AI, Ni J, Lau JS, Wilcox RA, Flies DB, Liu D, et al. B7-H3: a costimulatory molecule for T cell activation and IFN-gamma production. Nat Immunol. 2001; 2: 269-74.

24. Inamura $\mathrm{K}$, Amori $\mathrm{G}$, Yuasa $\mathrm{T}$, Yamamoto $\mathrm{S}$, Yonese $\mathrm{J}$, Ishikawa $\mathrm{Y}$. Relationship of B7-H3 expression in tumor cells and tumor vasculature with
FOXP3+ regulatory T cells in renal cell carcinoma. Cancer Manag Res. 2019; 11: 7021-30.

25. Zhang J, Zhang JK, Zhuo SH, Chen HB. Effect of a cancer vaccine prepared by fusions of hepatocarcinoma cells with dendritic cells. World J Gastroenterol. 2001; 7: 690-4

26. Shibolet O, Alper R, Zlotogarov L, Thalenfeld B, Engelhardt D, Rabbani E, et al. Suppression of hepatocellular carcinoma growth via oral immune regulation towards tumor-associated antigens is associated with increased NKT and CD8+ lymphocytes. Oncology. 2004; 66: 323-30.

27. Giusca SE, Wierzbicki PM, Amalinei C, Caruntu ID, Avadanei ER. Comparative analysis of CD4 and CD8 lymphocytes - evidences for different distribution in primary and secondary liver tumors. Folia Histochem Cytobiol. 2015; 53: 272-81.

28. Chen J, Jiang CC, Jin L, Zhang XD. Regulation of PD-L1: a novel role of pro-survival signalling in cancer. Ann Oncol. 2016; 27: 409-16.

29. Zheng S, Hedl M, Abraham C. TAM receptor-dependent regulation of SOCS3 and MAPKs contributes to proinflammatory cytokine downregulation following chronic NOD2 stimulation of human macrophages. J Immunol. 2015; 194: 1928-37.

30. Ruscica M, Ricci C, Macchi C, Magni P, Cristofani R, Liu J, et al. Suppressor of Cytokine Signaling-3 (SOCS-3) Induces Proprotein Convertase Subtilisin Kexin Type 9 (PCSK9) Expression in Hepatic HepG2 Cell Line. J Biol Chem. 2016; 291: 3508-19.

31. Yoneda T, Kunimura N, Kitagawa K, Fukui Y, Saito H, Narikiyo K, et al. Overexpression of SOCS3 mediated by adenovirus vector in mouse and human castration-resistant prostate cancer cells increases the sensitivity to NK cells in vitro and in vivo. Cancer Gene Ther. 2019; 26: 388-99.

32. Wang L, Kang FB, Shan BE. B7-H3-mediated tumor immunology: Friend or foe? Int J Cancer. 2014; 134: 2764-71. 